\title{
Shape Memory and Superelasticity Announces New Additions to the Editorial Advisory Board
}

It is with great privilege that Shape Memory and Superelasticiy announces the latest individuals to join the Editorial Advisory Board. Adding to the already exceptional list of members are the following outstanding individuals.

Ibrahim Karaman, Texas A\&M University, TX, USA Gunay Anlas, Bogazici University, Istanbul, Turkey Aaron Stebner, Colorado School of Mines, CO, USA (Effective September 2016)

Christian Lexcellent, University of Besancon, Besancon, France

Samantha Daly, University of Michigan, MI, USA Reginald Hamilton, Penn State University, PA, USA

Alan Pelton, G. Rau Inc., Santa Clara, CA, USA

Ken Gall, Duke University, Durham, NC, USA

The new members of the Editorial Advisory Board will be combined with those already serving who are listed below.

Brian T. Berg, Boston Scientific Corporation, Maple Grove, MN, USA

Eduard Cesari, University Illes Balears, Palma De Mallorca, Spain

Y. I. Chumlyakov, Tomsk State University, Tomsk, Russia

Alberto Coda, Saes Getters Spa, Milano, Italy

Franco Furgiuele, Univeristy of Calabria, Cosenza, Italy

David S. Grummon, East Lansing, MI, USA

Manfred Kohl, Karlsruhe Institute of Technology (KIT),

Karlsruhe, Germany

Yang Liu, Nanyang University, Singapore

Michael R. Mitchell, Mechanics \& Materials Consulting, LLC, Flagstaff, AZ, USA

Minoru Nishida, Kyushu University, Kasuga, Japan
Etienne Patoor, University of Metz, France

Sergey D. Prokoshkin, Moscow Institute of Steel and Alloys, Moscow, Russia

Robert O. Ritchie, University of California at Berkeley, Berkeley, CA, USA

Qing Ping Sun, Hong Kong University of Science \& Technology, Hong Kong, China

Ausonio Tuissi, CNR- IENI, Lecco, Italy

Jan Van Humbeeck, Catholic University Leuven, Leuven, Belgium

Ming H. Wu, Edward Lifesciences, Irvine, CA, USA

With the dedication of Shape Memory and Superelasticity's Editor, and Associate Editors listed below, along with the Editorial Advisory Board the journal will continue to provide our readers with articles of the highest quality.

Huseyin Sehitoglu, Editor, University of Illinois at Urbana-Champaign, Urbana, Illinois, USA

Tom Duerig, Associate Editor, Nitinol Devices \& Components, Inc., Fremont, CA, USA

Dimitris Lagoudas, Associate Editor, Texas A\&M University, TX, USA

Yinong Liu, Associate Editor, The University of Western Australia, Crawley, Australia

Hans Jurgen Maier, Associate Editor, Leibniz Universität Hannover, Garbsen, Germany

Shuichi Miyazaki, Associate Editor, University of Tsukuba, Tsukuba, Japan

Petr Sittner, Associate Editor, Academy of Sciences of the Czech Republic, Prague, Czech Republic

Thomas Waitz, Associate Editor, University of Vienna, Vienna, Austria 